Pure Appl. Chem., Vol. 73, No. 10, pp. 1599-1611, 2001.

(C) 2001 IUPAC

\title{
Selective detection of neurotransmitters by fluorescence and chemiluminescence imaging*
}

\author{
Ziqiang Wang and Edward S. Yeung ${ }^{\ddagger}$ \\ Ames Laboratory-USDOE and Department of Chemistry, lowa State University, \\ Ames, IA 50011, USA
}

\begin{abstract}
In recent years, luminescence imaging has been widely employed in neurochemical analysis. It has a number of advantages for the study of neuronal and other biological cells: (1) a particular molecular species or cellular constituent can be selectively visualized in the presence of a large excess of other species in a heterogeneous environment; (2) low concentration detection limits can be achieved because of the inherent sensitivity associated with fluorescence and chemiluminescence; (3) low excitation intensities can be used so that long-term observation can be realized while the viability of the specimen is preserved; and (4) excellent spatial resolution can be obtained with the light microscope so subcellular compartments can be identified. With good sensitivity, temporal and spatial resolution, the flux of ions and molecules and the distribution and dynamics of intracellular species can be measured in real time with specific luminescence probes, substrates, or with native fluorescence. A noninvasive detection scheme based on glutamate dehydrogenase (GDH) enzymatic assay combined with microscopy was developed to measure the glutamate release in cultured cells from the central nervous system (CNS). The enzyme reaction is very specific and sensitive. The detection limit with CCD imaging is down to $\mu \mathrm{M}$ levels of glutamate with reasonable response time. We also found that chemiluminescence associated with the ATP-dependent reaction between luciferase and luciferin can be used to image ATP at levels down to $10 \mathrm{nM}$ in the millisecond time scale. Similar imaging experiments should be feasible in a broad spectrum of biological systems.
\end{abstract}

\section{INTRODUCTION}

The interests and development of single-cell analytical techniques come from several sources. Conventional analysis tools are all bulk-average-based methods, which means the signal is taken from a collection of biological cells and the results are then estimated for a single cell. While this is acceptable for general biological analysis because individual variances in, for example, clinical samples fall into a broad range and do not cause problems in interpretation, it is not true for neurochemical analysis. The central nervous system (CNS) is a very complicated system with hundreds of different active molecules involved in numerous processes in different functional parts of the brain. It is also highly heterogeneous among the individual cells in the same functional part and even within the different parts of the same cell body. It is thus important to understand the variance for individual cells and the effect on physiological functions. The results from bulk-average methods will not be able to tell the real dynamics and mechanisms of the functions and pathways. Such is also important for clinical diagnostic purposes, because single cell analysis makes it possible to find the first few abnormal cells at their early

*Lecture presented at the IUPAC International Congress on Analytical Sciences 2001 (ICAS2001), Tokyo, Japan, 6-10 August 2001. Other presentations are published in this issue, pp. 1555-1623.

${ }^{\ddagger}$ Corresponding author 
stage of development. Otherwise, the overwhelming abundance of normal cells will likely mask them out if analyzed by bulk-average methods.

\section{SEPARATION AND SINGLE-CELL ANALYSIS}

The development of chromatographic microcolumn techniques, especially the progress in capillary electrophoresis (CE), makes it practically possible to advance single-cell analysis [1]. This is because: (1) the small dimension of the capillary format, in addition to the ease of manipulation of CE, makes sampling of single cells straightforward. The inner diameter of the capillary is usually only $10-50 \mu \mathrm{m}$, which is at the same dimension as that of cells; (2) the extremely high separation efficiency $\left(10^{5}-10^{6}\right.$ theoretical plates) expedites the separation of complex constituents from intracellular species when cells are lysed or caused to release material; (3) the capillary itself can be used as a reaction chamber after sampling, so various on-column derivatization procedures or treatments can be implemented to investigate cell functions and release kinetics of interest for selected reaction conditions.

Several kinds of neuronal cells have been studied by single-cell analysis techniques for the determination of neurotransmitters. Dopamine, serotonin, tyrosine, and tryptophan were identified and quantified from three different neurons, D2, E4, and F1, from the land snail helix aspersa by open tubular liquid chromatography (OTLC) with voltammetric detection [2]. The detection limit is at the femtomole level, which is much more sensitive than conventional methods. The advantage of this method comes from the high resolving power from small columns and the high sensitivity of electrochemical detectors.

Catecholamines (norepinephrine and epinephrine) in single bovine adrenal medullary cells were determined quantitatively by $\mathrm{C}-18$ reverse-phase microcolumn liquid chromatography [3]. With an electrochemical detector, the detection limit is down to tens of attomoles. Through analysis of over 20 individual cell samples, the variance of catecholamines among these cells was demonstrated and their physiological implications were discussed.

More progress was achieved by exploiting capillary electrophoresis. The common procedure is that a single cell is injected into the front end of the capillary, in which the sample volume is only subnanoliter with a 10-20 $\mu \mathrm{m}$ i.d. capillary. Either on-column derivatization in the sample zone followed by separation or post-column derivatization after separation is carried out. The detection is achieved by various methods such as electrochemical, laser-induced fluorescence, and immunoassay [4-6]. Compared to precolumn derivatization commonly adopted in microcolumn liquid chromatography [7], on-column and post-column derivatization is easier to implement in CE for the detection of molecules that cannot fluoresce naturally. This is because of the ease of manipulation in the CE format to carry out derivatization on column and, more importantly, the lack of sample dilution due to the size of the capillary.

Hemoglobin and carbonic anhydrase in individual erythrocytes have been separated by CE and quantified by using laser-induced fluorescence (LIF) detection with a UV line from an Ar ion laser [8]. The activities of several lactate dehydrogenase isoenzymes have been determined in individual human erythrocytes by monitoring the enzyme-catalyzed production of NADH using LIF detection after separation of the isoenzymes [9]. Exocytotic release of serotonin, insulin, and catecholamine from single mast cells, pancreatic $\beta$-cells, and adrenal chromaffin cells were quantitatively monitored by CE-LIF [10-12]. An on-column derivatization procedure with LIF detection by CE was developed, and the analysis of individual rat pheochromocytoma (PC12) cells was demonstrated. Dopamine and 5 amino acids were detected and determined quantitatively [13].

The development of these CE-based methods demonstrates that CE is well suited for the analysis of a wide variety of molecules found in cells, and is a powerful tool for dealing with the heterogeneity and complexity in neurochemical analysis. 


\section{FLUORESCENCE MICROSCOPIC METHODS}

Fluorescence microscopy is a powerful tool in that it allows spatial and temporal visualization of fluorescent materials in microscopic specimens. Because of this capability, this technology has been used in neuroscience to address important fundamental physiological functions and dynamics. It allows users to look inside the neuronal cells and brain tissue slices with exceptional detail. It allows the acquisition of spatial information and temporal alterations of components that are either intrinsically fluorescent or that have been coupled to extrinsic fluorescent molecules. The combination of the specificity inherent in current fluorescence techniques with the sensitivity of fluorescence imaging systems has led to the ability to detect very small amounts of material with very high sensitivity and precision.

Various applications have been developed in neurochemical analysis by using fluorescence imaging techniques. Immunofluorescence, which can be used for the identification, localization, and visualization of cell and tissue components, is based on the fact that it is possible to produce highly specific antibodies against individual cell constituents (antigens), to label these antibodies with fluorescent molecules and then visualize these fluorescent antibodies after they have been applied to microscopic specimens. Fluorescence in situ hybridization (FISH) is similar to immunofluorescence except that FISH allows the direct visualization and localization of DNA and RNA sequences on chromosomes in cells and in tissues. Digitized video microscopy, in which the physiological functions of intact cells and tissues are manipulated and recorded in sequential images, can show multiple cellular activities in sample specimens in real time. In various studies, the kinetic processes in living cells, genetic variations of DNA and RNA inside cell chromosomes, and 2-D and 3-D characteristic cell image reconstruction have all been intensively explored with fluorescence microscopy techniques.

Many important biological molecular constituents and free ions (such as $\mathrm{H}^{+}$and $\mathrm{Ca}^{2+}$ ) relevant to signal transduction processes have been studied with fluorescence microscopy. Fluorescent labeling probes for target molecules or native fluorescence methods have been developed, and excellent results for molecular distribution and dynamics of processes such as endocytosis and excytosis have been obtained.

Calcium imaging in neuronal cells is a well-established method to monitor the distribution and dynamics of calcium ion movement in cells. With the use of calcium sensitive dyes (e.g., fura-2 and fluo-3), the concentration changes of calcium ions in different kinds of neurons and glial cells [14-18] have been successfully exploited, and great progress has been achieved with respect to various pathways in which calcium ions are involved to provide understanding of the nature of activities in the brain.

Serotonin is another important neurotransmitter involved in many endocytosis and exocytosis processes in CNS. Because serotonin possesses native fluorescence, the LIF method has been developed with the advantages of specificity, low background, and high sensitivity. The uptake of serotonin by single astrocytes was first monitored by LIF microscopy with simultaneous temporal and spatial resolution [19]. The exocytotic release of serotonin from astrocytes, mast cells was also studied with LIF microscopy [20,21]. The highly localized phenomenon of granule and vesicle release dynamics is revealed with the help of the high spatial and temporal resolution of fluorescence microscopy.

\section{GLUTAMATE IN THE CENTRAL NERVOUS SYSTEM}

Five amino acids have been considered to serve neurotransmitter functions in the central nervous system (CNS). Among these five compounds, glutamate and aspartate are considered to play excitatory function and $\gamma$-aminobutyric acid (GABA), glycine, and taurine are believed to work as inhibitory neurotransmitters [22]. Glutamate is considered as a major excitatory neurotransmitter compared to aspartate [22]. The possibility of glutamate serving as an active excitatory neurotransmitter in CNS was first demonstrated in the 1950s [23-25]. It is found that glutamate could stimulate depolarization in the spinal cord at the single neuron level [24,25]. Initially, scientists could not accept the implication of glutamate as a neurotransmitter because of the high concentration levels and wide distribution of glutamate 
in CNS. Glutamate is found existing in the cortex around $80 \mu \mathrm{M} / \mathrm{g}$ protein [26,27] and in synaptic vesicles as high as $60 \mathrm{mM} / \mathrm{g}$ protein $[28,29]$. On the other hand, the identified neurotransmitters at that time, acetylcholine and catecholamine, both exist in very restricted regions in CNS and at low concentrations.

Nevertheless, evidence was established to support the fact that glutamate is an excitatory neurotransmitter in the peripheral and CNS in the late 1970s and 1980s [30-32]. In 1965, it was first reported [33] that glutamate is released from the cortex of cats with the surface superfusion approach. It is also found that the release of glutamate was accompanied by the release of aspartate, glycine, and taurine after electrical stimulation. In 1976, it is observed that the release of glutamate from the visual cortex of rats by electrical or high $\mathrm{K}^{+}$stimulation was $\mathrm{Ca}^{2+}$ dependent [34].

However, there was no evidence that the sites of glutamate release were in the neural circuits of the cortex at that time. Later in 1985, it was discovered that the ipsilateral cortico-cortical association fiber releases glutamate as a transmitter [35]. Around that time, several regions were also found to release glutamate, such as the collosal projection fibers and the neostriatum in a moving rats [36,37]. This led to the discovery of the spatial discrimination of glutamate release. There are five classes of synaptic receptors that have been identified for glutamate, each of which is named for a selective agonist: $N$-methyl-D-aspartate (NMDA), kainate (KA), AMPA, ACPD, and AP4 [38-41].

In glial cells, glutamine is generated from either the intake of released glutamate from neurons or from glutamate generated from the citric acid cycle (TCA) in the presence of glutamine synthetase. Glutamine is then discharged, along with another component from TCA, $\alpha$-ketoglutarate, from the glial cells into extracellular space and enters the nerve terminal of presynaptic neurons by a low affinity system or diffusion. There, the glutamate is converted from glutamine by glutaminase and from $\alpha$-ketoglutarate by glutamic dehydrogenase (GDH). The glutamate then has three pathways: the first is to move to a transmission pool ready for the synaptic transduction cycle, the second functions as the precursor of GABA, and the rest goes to a metabolic pool. The released glutamate from presynaptic cells into extracellular space will be absorbed by postsynaptic cells to complete the signal transduction process, converted to GABA or glutamine there, and transferred to glial cells along with the excess amount of glutamate left in cleft. The cycle then continues to the next round.

The glutamate transmitter system has captured the attention of a wide range of investigators because it not only mediates standard fast excitatory synaptic transmission, via ionotropic receptors, but also participates in more complex neuronal process, such as development, learning and memory, and even neuropathology. The capacity of glutamate to contribute to these processes is a result of the wide variety of intercellular signals that this transmitter can generate and thus interact with other neurons and glial cells through receptor-mediation systems. On the other hand, overstimulation of these signaling processes by glutamate could also cause neuronal damage associated with a wide variety of neurological insults and diseases, including ischemia, anoxia, stroke, hypoglycemia, epilepsy, Huntington's disease, amyotrophic lateral sclerosis, lathyrisms, and Alzheimer's disease.

For studying the role of glutamate behavior in signal transduction, there is no doubt that real-time imaging of glutamate movement and concentration change will greatly enhance the understanding of many important neuronal activities. Though some efforts have been made such as the simulation of realtime release dynamics of glutamate between pre- and postsynaptic cells [42], no real imaging data was available. The difficulties lie in the fact that the whole process of synaptic glutamate release may be over in a few milliseconds at extremely small dimensions (the cleft between pre- and postsynaptic cells is usually only several hundred nanometers in size). Also, glutamate has no inherently fluorescent groups, and there is no effective derivatization method established to detect such fast, transient processes.

Based on the characteristics of CNS, it is imperative for any successful analysis method to have following capabilities:

- High selectivity. The CNS is a complicated network system formed by many types of cells. With so many active processes present at the same time, the method ought to be able to distinguish analyte molecules from the thousands of possible interfering factors. 
- High sensitivity. The concentrations of neurotransmitters present both in intracellular and extracellular fluids needed to perform normal neurotransmitter functions are usually very low, typically around $\mu \mathrm{M}$ or even down to $\mathrm{nM}$.

- $\quad$ Fast response. The dynamic signal transduction process for neurotransmitters could be initiated and completed in a few milliseconds. Under this time scale, any method that cannot respond fast enough would not be able to reveal the true behavior of neurotransmitters in CNS.

- Miniaturization. The dimensions of neurons and glial cells in CNS are only at micrometer size, and the distance between the pre- and postsynaptic cells is at the nanometer range. In order to obtain representative data of neurotransmitters at good spatial resolution for such extremely heterogeneous systems, the sampling ability of the method should be able to match such dimensions.

The techniques used to evoke glutamate release include ionophores, electrical stimulation and high concentrations of $\mathrm{K}^{+}$. The first method applies certain compounds to the cell membrane to open the voltage-sensitive ion channels such as sodium, potassium, and calcium channels. The intracellular concentrations of such ions thus increase and cause the cell membrane to depolarize. As a consequence, the glutamate ion is released with other ionic species through the ion channels of the cell membrane. The side effect is that such compounds may cause cell damage after depolarization $[43,44]$.

Applying electrical pulses to the neuron membrane is another technique to evoke glutamate release. When the electrical current passes the external solution medium, a small fraction of it would pass across the cell membrane, and thus some voltage-dependent channels open and cause the depolarization of the cell [45].

The last method to evoke glutamate release is to raise the external $\mathrm{K}^{+}$concentration. This method is the most popular one among the three techniques owing to the ease of use. The $\mathrm{K}^{+}$concentration required for stimulation ranges from $10-80 \mathrm{mM}$, while the normal concentration is about $2-4 \mathrm{mM}$. Upon such an increase of $\mathrm{K}^{+}$concentration, the calcium channel in the cell membrane will open and the intracellular calcium concentration will increase. This triggers the exocytosis process, and the content of glutamate pools in the synapse area will be released. So, this type of synaptic release of glutamate is calcium-dependent [46].

The collection of released glutamate is generally done by sample perfusions. Two types of techniques are usually used and, based on their collection mode, they could be categorized as "static" and "dynamic" modes. In the first mode, the sample tissue is incubated in a neurophysiological medium, such as Ringer's solution, and is stimulated by one of the methods previously described. Then, the solution containing the released glutamate is collected and analyzed [47]. This method can be used for "in situ"-type experiments. Complementary to this method, the second technique is aimed to collect glutamate released on a continuous basis. In this method, small fractions of samples are continuously collected through specific devices during the entire stimulation period. In this way, the dynamics of cell release of glutamate can be studied. The most widely used device is a microdialysis tubing. A microdialysis tubing probe is inserted into the sampling region, such as a specific area of tissue slices or cleft areas between different brain parts. The carrier medium is flowed to the probe sensing tip and back so that glutamate in the sensing region outside the tip can be collected into the carrier medium based on differential concentration inside and outside the tube. By using different types of dialysis tubing, species with high molecular weight can be included or excluded. In addition, the probe is generally flexible and can be applied directly to the location of interest because it will not damage the tissue. This method can be used for "in vivo"-type experiments [48].

HPLC is the most common technique for amino acid determinations. Numerous methods have been investigated for free amino acid determinations, which mainly apply either pre- or postcolumn derivatization with ultraviolet, fluorescence, or electrochemical detection schemes [49-54]. Among these methods, separation on a reverse-phase column followed by fluorescence or electrochemical detection provides the most sensitive and simplest methodology. The separation is fast, and good resolution is obtained with gradient systems. In addition, almost all of the amino acids can be separated and 
measured at the same time. The only disadvantage is that the gradient system is time-consuming because of the need to re-equilibrate the column between runs.

The $o$-phthaldialdehyde (OPA) derivatization reaction is commonly used in precolumn derivatization with fluorescence or electrochemical detection. The OPA reagent reacts with amino acids rapidly to generate a highly fluorescent compound, thio-substituted isoindoles [55]. The mobile phase includes methanol + buffer system such as acetate, phosphate at acidic $\mathrm{pH}$, and with a gradient system to reduce the retention time and stabilize the derivatives. The retention time for amino acid neurotransmitters is around 2-12 min with this method. The detection limit is at about the subpicomole range.

Gas chromatography-mass spectrometry (GC/MS) is a powerful tool for amino acid analysis. With derivatization on the GC column, a detection limit down to 10 attomoles could be achieved [56]. Because fragmentation occurs during MS, prepurification of the sample is necessary to exclude interference ions to guarantee maximum selectivity.

Isotope assays involve treating the amino acids with a radiolabeled compound. The resulting radiolabeled amino acids are detected by various assays [57]. Radioreceptor assays are commonly used for amino acid neurotransmitters. In this procedure, the radiolabeled components are incubated with the tissue that contains the neurotransmitter receptor of interest and the ligand binding to specific recognition sites is examined. Another assay is based on the double-isotope dansyl microassay [58]. The amino acid sample is added to a mixture of ${ }^{14} \mathrm{C}$-labeled amino acids, and the compound is dansylated by $\left[{ }^{3} \mathrm{H}\right]$-dansyl chloride. The dansylated amino acids are then separated by thin-layer chromatography and the ratio between $\left[{ }^{3} \mathrm{H}\right]$ and $\left[{ }^{14} \mathrm{C}\right]$ for each dansyl amino acid is measured. The ratio shows a linear relationship to the unlabeled amino acid in the original sample.

Enzymatic assays are based on using an enzyme that is selective to the specific analyte and measuring the concentration of the assay products by various detection methods, which in turn can be related back to the analyte concentration. The enzymes that are selective to glutamate include glutamate dehydrogenase, glutamate oxidase, and glutamate decarboxylase. The common procedure used is to add the enzyme and required cosubstrates to the sample and observe the signal generated from the reaction products by means of fluorescence, luminescence, or spectrophotometry.

Various schemes for measuring glutamate based on enzymatic assay have been developed [59-67]. With the utilization of glutamate dehydrogenase, coupled with cosubstrate $\mathrm{NAD}^{+}$, the product of the reaction, NADH, can be monitored either by fluorescence or by absorption. The signal of NADH generated then can be used to calculate the glutamate concentration. For assays utilizing glutamate oxidase, the reaction includes consumption of oxygen and generation of ammonia and hydrogen peroxide. The measurements are thus performed by the detection of either the depletion rate of oxygen in the reaction medium or the generation rate of ammonia and peroxide. Several oxygen and ammonia gas sensors were successfully built with this principle and applied to neurochemical analysis. In addition, the peroxide generated in the reaction can be further coupled to other peroxidase-based enzyme assays, and the final products can be monitored by fluorescence or chemiluminescence. Glutamate decarboxylase catalyzes the reaction of glutamate in the presence of water to generate $\gamma$-aminobutyric acid and carbon dioxide. Detection is accomplished either by monitoring the generation rate of carbon dioxide with a gas sensor or by coupling to a $\mathrm{pH}$-sensitive dye, since the accumulation of carbon dioxide in the reaction solution can cause $\mathrm{pH}$ changes in a certain range. The signal from the dye can be measured spectrophotometrically.

Enzymatic assays are fast and sensitive. They usually have a wide range of linear response for measured glutamate. The inherent high selectivity eliminates time-consuming sample pretreatment steps aimed to exclude interfering factors, and thereby offers a simple, convenient, and reliable approach for amino acid determination. The only disadvantage is that the enzymes used are usually expensive. The conventional format of addition of the enzyme mixture to the sample solution makes it impossible for the enzymes to be reused and is thus not cost-effective. This hinders the wide application of this approach in neurochemical analysis. The compromise approach prevalent today is to immobilize the enzyme onto a solid support in the form of a sensor. Then, the enzyme can be more stable and 
can be used repeatedly. Although this approach comes along with a certain degree of sacrifice of sensitivity from the immobilization procedure due to denaturation of the enzyme, it has been demonstrated that the biosensor format offers advantages on stability, ease of manipulation, signal reproducibility, and, of course, cost effectiveness.

A noninvasive detection scheme based on glutamate dehydrogenase enzymatic assay combined with microscopy was developed by us to measure the glutamate release in cultured cells from the central nervous system (CNS) [68]. The enzyme reaction is very specific and sensitive. The detection limit with CCD imaging is down to $\mu \mathrm{M}$ levels of glutamate with reasonable response time ( $30 \mathrm{~s})$. The standard glutamate test shows a linear response over 3 orders of magnitude, from $\mu \mathrm{M}$ to $0.1 \mathrm{mM}$ range. The in vitro monitoring of glutamate release from cultured neuron cells demonstrates excellent spatial and temporal resolution.

In this study, we used a dual-enzyme reaction theme as that reported earlier $[68,69]$ for the analysis of glutamate in single cells by capillary electrophoresis:

Glutamate $+\mathrm{NAD}^{+} \rightarrow \alpha$-ketoglutarate $+\mathrm{NH}_{4+}+\mathrm{NADH}$
$($ GDH catalyzed $)$

$\alpha$-ketoglutarate + alanine $\rightarrow$ pyruvate + glutamate

(GPT catalyzed)

Besides enhancing the specificity of the reaction, the introduction of the second enzyme, glutamic pyruvate transaminase, greatly enhances the reaction efficiency by taking up $\alpha$-ketoglutarate to drive the reaction toward NADH generation. The reaction rate becomes much faster compared to conventional single-GDH-enzyme reaction as reported in the literature [2,3]. Consistent with what we found in the CE experiments, it was necessary to have a large excess of cosubstrates $\mathrm{NAD}^{+}$and L-alanine to accelerate the reaction to assure that the CCD signal is linear with initial glutamate concentration. The choice of 305-nm excitation is guided by the need to limit the background fluorescence from $\mathrm{NAD}^{+}$. Under our experimental conditions, we were able to obtain satisfactory NADH fluorescence images at $460 \mathrm{~nm}$ over the course of $1 \mathrm{~min}$ after injection for initial glutamate concentrations down to $1 \mu \mathrm{M}$ and for CCD exposure times at $500 \mathrm{~ms}$ per frame.

Figure 1 shows the typical ( $n=20$ experiments) fluorescence images of NADH generated from the glutamate enzyme reaction. All fluorescence images are the result of subtraction of the final frame (1 min after stimulation) from the initial frame (before stimulation) after background correction but with no other modifications. By this flat-fielding method, all contributing interference signals such as

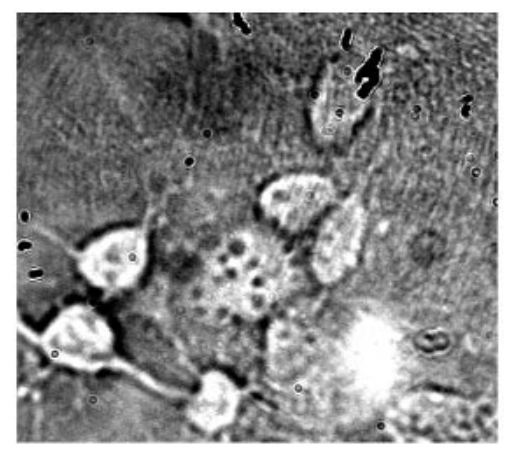

A

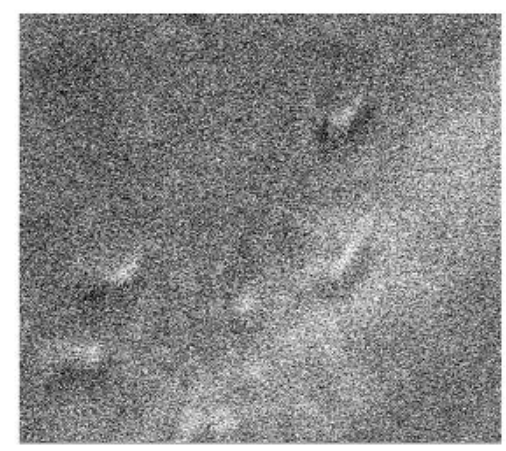

B

Fig. 1 Glutamate release from a single neuron in relatively isolated environment. A, optical image of the neuron and $\mathrm{B}$, corresponding fluorescence image. The exposure time is set at $500 \mathrm{~ms}$ per frame. Frames of fluorescence image were taken sequentially during and after stimulation. The image shown was the average of 5 consecutive frames. The imaged area is $100 \times 100 \mu \mathrm{m}$. 
fluorescence from background matrix, cell membranes and cell nuclei, basal release of glutamate, and stray light were canceled out. The NADH generated is the result of enzyme reaction from released glutamate from neurons due to high- $\mathrm{K}^{+}$stimulation, because control experiments at normal potassium concentrations gave no detectable signals.

From these images, it can be seen that although glutamate is released all over the cell body area, only some parts of the cell, mostly along the edges of the cell, have strong release and are thus represented by bright areas of NADH fluorescence. It is believed that in CNS the structure of the neuronal network consists of dendrite and axon fibers extended from neuron bodies to neighboring neurons and glial cells, near and far away, covering each other and forming signaling patch pairs. At the ends under the buttons from the fibers there are nerve terminals that form the pre- and postsynaptic terminals. Neurotransmitters are released from presynaptic terminals, upon stimulation, into the cleavage space between the synapse pair and move to postsynaptic terminals to activate specific receptor groups to complete the signal transduction process. Though glutamate is spread all over the neuronal cell compartments, the average concentration is relatively low. Only glutamate released from synapses due to stimulation, in the form of vesicles (and granules) expelled mainly through exocytosis (due to high- $\mathrm{K}^{+}$ stimulation in our case) would have temporarily localized concentrations that are elevated. This high concentration of glutamate would also quickly decrease to the low level of extracellular concentration due to dilution through diffusion and absorption by postsynaptic terminals to complete signal transmission and to protect cells from exocytotoxicity. This fast phenomenon is the major reason that the direct monitoring of glutamate release in CNS is difficult. However, this behavior favors observation of the signal derived from the first quanta of release on stimulation with elevated potassium concentration. This is because as long as glutamate is released rapidly, only the localized high concentration of glutamate would be captured by transient reaction with the enzyme mixture to generate enough NADH for detection. It should be noted that diffusion limits the spatial resolution here, and submicron resolution is not possible.

\section{ATP IN CENTRAL NERVOUS SYSTEM}

Adenosine-3"-triphosphate (ATP) is best known as the most important energy source in cellular metabolism. Almost all living cells contain ATP. It plays a central role in the energy status of the cell because it serves as the energy source for all kinds of metabolic changes and cellular functioning [70,71]. In addition, ATP is also found to have regulation ability for intracellular enzyme activity $[72,73]$.

Several analytical techniques have been developed for the determination of ATP in biological samples. Electrochemical-based methods, cyclic voltammetry, and chronoamperometry are used for ATP measurements based on competitive receptor binding with hexacyanoferrate(II) ion because the ATP-receptor binding is nonelectroactive [74]. Theoretical study shows that the sub-millimolar concentrations of ATP could be detected. Also, sinusoidal voltammetry has been successfully developed to directly measure ATP concentration, and a detection limit of submicromolar levels can be reached with this technique [75]. It was reported [76] that rapid Fourier transform infrared (FTIR) spectroscopy and time-resolved single-wavelength infrared spectroscopy (IR) have been used to follow the photochemical release of ATP. The temporal resolution of these IR techniques can be around the millisecond-tosecond range, although biological applications have not been developed yet.

The most widely used technique in biological science so far for the determination of ATP is based on the firefly luciferase-luciferin bioluminescence assay [77-85]. In this assay, the enzyme extract from firefly photinus pyralis, luciferase, is coupled to cosubstrates luciferin (D-form), and, in the presence of magnesium ions, catalyzes the conversion of ATP to AMP and pyrophosphate. Accompanying the products of the reaction is light emission with wavelength around $560 \mathrm{~nm}$, as depicted in the following equations:

$$
\mathrm{E}+\mathrm{LH}_{2} \stackrel{\mathrm{Mg}}{\longrightarrow} \mathrm{E}-\mathrm{LH}_{2}+\mathrm{ATP} \longrightarrow \mathrm{E}-\mathrm{L}-\mathrm{AMP}+\mathrm{PP}
$$




$$
\mathrm{E}-\mathrm{L}-\mathrm{AMP} \stackrel{\mathrm{O}_{2}}{\longrightarrow} \mathrm{E}-\mathrm{L}+\mathrm{AMP}+\mathrm{h} v(560 \mathrm{~nm})
$$

E: luciferase; $\mathrm{LH}_{2}$ : D-luciferin; PP: pyrophosphate; L: deoxyluciferin.

This reaction is fast, specific, and sensitive. After only a few hundred milliseconds upon enzyme coupling to the ATP molecule, light output can be observed. The specificity of the enzyme is excellent: only ATP can trigger the light emission reaction. Many analogs of ATP such as ADP, AMP, UDP, and other purine nucleotides have no effect on the enzyme, so interference can be controlled. The reaction is also very sensitive: with the right combination of enzyme and substrate concentrations and proper instrument conditions, a total amount down to attomoles of ATP from extracts of cell samples can be detected.

The typical light output curve from this reaction is shown in Fig. 2 [69]. Through careful studies, it was found that the peak maximum can be used to quantitatively determine the amount of ATP in the reaction. The linear relationship is excellent $(R=0.999)$, and the dynamic response ranges over 4 to 5 magnitudes starting from $10^{-18} \mathrm{~mol}$, even with commercial assay kits without any modifications.

Various applications of this assay in biological studies have been reported [86-93]. The determinations of ATP content in different types of cells and tissues were used in clinical diagnostics, such as bacteria testing and antibiotic assay. It is also used in microbiology, food industry, pharmaceuticals, environmental monitoring, and even space technology.

In the 1970s, ATP was first found to have an effect on neurotransmission in some CNS systems [94], although there was skepticism for ATP as a neurotransmitter or neuromodulator back then. In the last 20 years, critical evidence for ATP as a neurotransmitter or neuromodulator has been found, and the role of ATP in CNS is confirmed [95-99].

It is therefore very interesting and important to monitor ATP as a neurotransmitter in CNS at the cellular and subcellular level. The release of ATP from the micro-dissected organ of Corti has been measured with a bioluminescent assay [100]. The extracellular concentration of ATP in hippocampal slices from seizure-prone mice has been estimated [101]. Coreleases of ATP with some other wellknown neurotransmitters such as norepinephrine and acetylcholine were discovered and studied by electrophysiological methods [102-106]. The various groups of ATP-specific receptors have been identified and classificated [107].

A novel chemiluminescence dynamic imaging method was developed to monitor ATP release from living biological cells [69]. The detectability of ATP is down to $10^{-8} \mathrm{M}$ at millisecond exposure times with an intensified charge-coupled device camera. The direct imaging of ATP waves in astrocyte

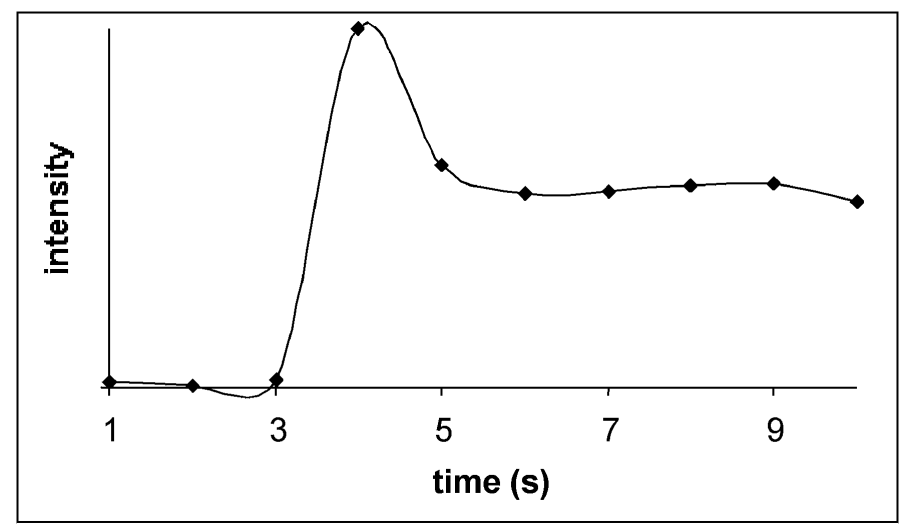

Fig. 2 Light emission response to ATP from the luciferase-luciferin reaction.

(C) 2001 IUPAC, Pure and Applied Chemistry 73, 1599-1611 
cultures was performed together with fluo-3-Ca imaging at millisecond temporal resolution and $\mu \mathrm{m}$ scale spatial resolution.

Confluent astrocytes [108] were stimulated mechanically via microtips. While not a natural stimulus, mechanical stimuli provide a high degree of spatio-temporal control that mimics the action of physiological ligands [3]. Consistent with the extracellular signaling hypothesis, physical contact, which is known to cause a calcium wave, immediately generated an extracellular ATP signal. As soon as an ATP signal appeared, the tip was withdrawn vertically. Extracellular diffusion during exposure limits the spatial resolution of these ATP images to $10 \mu \mathrm{m}$. Consistent with previous observations of calcium wave propagation [61], the average effective ATP wave excursion distance is $258 \pm 50 \mathrm{~mm}$ $(n=25)$. This argues against the single point release model, in which the effective signal travel distance is $110 \pm 30 \mathrm{~mm}[7,109]$, and implies distinct contributions from cells along the path. The existence of a finite propagation range for the ATP wave, plus the fact that the intensities decrease gradually along the path, support the postulation $[110,111]$ that subpopulations of astrocytes express different specific purinergic receptor subtypes. This dictates that not all astrocytes have the same sensitivity to ATP and they do not release identical amounts of ATP when stimulated. From the injection of standard ATP solution, the concentration of ATP released into the extracellular fluid can be estimated to be at the $\mu \mathrm{M}$ range, which agrees with previous reports $[48,108]$. The basal release is not detectable. Since intracellular ATP concentrations are in the $\mathrm{mM}$ range, the present reporter scheme cannot detect those changes that result from stimulation.

ATP is known to evoke intercellular calcium wave propagation from glial [26,112] and other [50] cells. The astrocyte cultures were loaded with fluo-3 [60], and the fluorescence on binding with intracellular $\mathrm{Ca},\left[\mathrm{Ca}^{2+}\right]$, was monitored simultaneously with the chemiluminescence signal from the released ATP. On stimulation, the intercellular calcium wave is found to be synchronized spatially and temporally with the extracellular ATP wave (Fig. 3). An important feature is that $\left[\mathrm{Ca}^{2+}\right]$ (Fig. 3b) persists at an elevated level for some time after stimulation while the ATP level (Fig. 3a) decreases soon after stimulation. This is an inherent difference between intracellular components that are trapped and extracellular components that can diffuse away. Also, there is a dramatic decrease in the ATP signal but only a modest decrease in the $\left[\mathrm{Ca}^{2+}\right]$ signal as a function of distance from the point of stimulation (Fig. 3). Yet, the calcium wave does not propagate beyond the excursion range of the ATP wave. This suggests that the two waves are not related to each other in a straightforward manner.

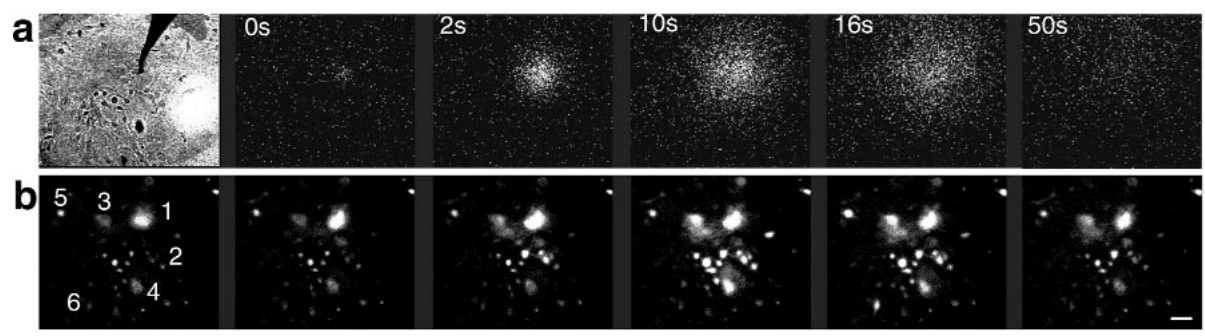

Fig. 3 Simultaneous monitoring of ATP and $\left[\mathrm{Ca}^{2+}\right]$ from astrocyte cultures showing that the calcium wave is synchronized with the released ATP upon stimulation. The exposure time for ATP is $500 \mathrm{~ms}$ and that for $\left[\mathrm{Ca}^{2+}\right]$ is 50 ms. a, bright-field image showing the position of the stimulation tip followed by ATP images. Times are relative to the time of stimulation. $b$, corresponding calcium images taken at $0.5 \mathrm{~s}$ delay relative to each ATP image shown above it. Cell 1 is at the position of stimulation. Initially, only the calcium level in cell 1 increased. As the experiment progresses, cells 2 and 3, then cells 4 and 5, and finally cell 6 showed increases in calcium level synchronized with the arrival of the ATP wave shown in a. The last image showed calcium levels falling back to normal coinciding with the fading of the ATP wave. 


\section{SUMMARY}

The ability to image neurotransmitters at the subsecond range and at micromolar concentration levels has led to elucidation of important signaling pathways. The development of laser excitation, labeling fluorophors, enzymatic reactions, and sensitive array detectors has made such experiments possible. It is obvious that sensitive and selective biosensors will continue to play an important role as a bridge between analytical chemistry and biology.

\section{ACKNOWLEDGMENT}

The Ames Laboratory is operated for the U.S. Department of Energy by Iowa State University under Contract No. W-7405-Eng-82. This work was supported by the Director of Science, Office of Basic Energy Sciences, Division of Chemical Sciences.

\section{REFERENCES}

1. E. S. Yeung. J. Chromatogr. A 830, 243-262 (1999).

2. R. T. Kennedy and J. W. Jorgenson. Anal. Chem. 61, 436-441 (1989).

3. B. R. Cooper, J. A. Jankowski, D. J. Leszczyszyn, M. R. Wightman, J. W. Jorgenson. Anal. Chem. 64, 691-694 (1992).

4. A. Ewing, J. M. Mesaros, P. F. Gavin. Anal. Chem. 66, 527A-537A (1994).

5. E. S. Yeung. Adv. Chromatogr. 35, 1-51 (1995).

6. F. E. Regnier, D. H. Patterson, B. J. Harmon. Trends Anal. Chem. 14, 177-181 (1995).

7. R. T. Kennedy, M. D. Oates, B. R. Cooper, B. Nickerson, J. W. Jorgenson. Science 246, 57-63 (1989).

8. T. T. Lee and E. S. Yeung. Anal. Chem. 64, 3045-3051 (1992).

9. Q. Xue and E. S. Yeung. Anal. Chem. 66, 1175-1178 (1994).

10. S. J. Lillard, E. S. Yeung, M. A. McCloskey. Anal. Chem. 68, 2897-2904 (1996).

11. W. Tong and E. S. Yeung. J. Chromatogr. B 689, 321-325 (1997).

12. W. Tong, E. S. Yeung. J. Neurosci. Meth. 82, 151-158 (1998).

13. S. D. Gilman and A. G. Ewing. Anal. Chem. 67, 58-64 (1995).

14. D. A. Williams and F. S. Fay. J. Biophys. 47, 131A-139A (1985).

15. T. Murphy, J. Baraban, W. Wier, L. Blatter. Science 263, 529-532 (1994).

16. A. Cornell-Bell, S. Finkbeiner, M. Cooper, S. Smith. Science 247, 470-473 (1990).

17. A. Pol, J. Wuarin, F. Dudek. Science 250, 1276-1278 (1990).

18. D. Jovin, M. Nicoud, S. Kaufman, T. Jovin. Science 230, 247-256 (1985).

19. W. Tan, V. Parpura, P. G. Haydon, E. S. Yeung. Anal. Chem. 67, 2575-2579 (1995).

20. S. J. Lillard and E. S. Yeung. J. Neurosci. Methods 75, 103-109 (1997).

21. W. Tan, P. G. Haydon, E. S. Yeung. Appl. Spectrosc. 51, 1139-1143 (1997).

22. J. P. Christopher. Surv. Ophthalmol. 29(5), 355-365 (1985).

23. T. Hayashi. Keio J. Med. 302, 183-188 (1954).

24. D. R. Curtis and R. M. Eccles. J. Physiol. (London) 141, 435-445 (1958).

25. D. R. Curtis, J. W. Philis, J. C. Watkins. Nature 183, 611-612 (1959).

26. M. Banay-Schwartz, A. Lajtha, M. Palkovitz. Neurochem. Res. 373, 252-257 (1986).

27. M. Palkovitz, T. Lang, A. Patthy, I. Elekes. Brain Res. 373, 252-258 (1986).

28. P. M. Burgar, E. Mehl, P. L. Cameron, P. R. Maycox, M. Baumert, F. Lottspeich, P. D. De Cammili, R. Jahn. Neuron 3, 715-720 (1989).

29. S. Naito and T. Ueda. J. Neurochem. 44, 99-105 (1985).

30. G. E. Fagg and A. C. Foster. Neurosci. 9, 701-719 (1983).

31. F. Fonnom. J. Neurochem. 42, 1-11 (1984). 
32. P. Roberts, J. Storm-Mathisen, G. Johnston. Glutamate: Transmitter in the Central Nervous System, Wiley, New York (1981).

33. H. H. Jasper, R. T. Khan, K. A. Elliott. Science 147, 1448-1449 (1965).

34. R. Clark and G. Collins. J. Physiol. (London) 263, 383-403 (1976).

35. T. P. Hicks, R. W. Guedes, W. L. Veale, J. Veenhuizen. Exp. Brain. Res. 58, 421-425 (1985).

36. D. Druce, D. Peterson, J. De Belleronche, H. F. Bradford. Brain. Res. 247, 303-307 (1982).

37. J. Peinado, M. Iribar, R. Myers. Neurochem. Res. 12, 629-634 (1987).

38. M. Mayer and G. Westbrook. Prog. Neurobiol. 28, 197-276 (1987).

39. T. Tadaharu. Neurosci. Res. 9, 79-82 (1990).

40. H. Bradford. Chemical Neurobiology: An Introduction to Neurochemistry, W. H. Freeman, New York (1986).

41. G. Fagg. TINS May (1985).

42. A. R. Dmitri and M. K. Dimitri. J. Neurosci. 18, 3158-3170 (1998).

43. W. Walz. In Neuromethods 3: Amino Acids, A. Boulton and G. Baker (Eds.), Humana Press, Clefton, NJ (1985).

44. D. Nicholls and D. Attwell. TiPS 11, 462-468 (1990).

45. A. Greenshaw. In Neuromethods 3: Amino Acids, A. Boulton and G. Baker (Eds.), Humana Press, Clefton, NJ (1985).

46. P. Lipton. In Neuromethods 3: Amino Acids, A. Boulton and G. Baker (Eds.), Humana Press, Clefton, NJ (1985).

47. V. Parpura, F. Liu, S. Brethorst, K. Jeftinija, S. Jeftinija, P. G. Haydon. FEBS Letters 360, 266-270 (1995).

48. U. Ungerstedt. In Measurement of Neurotransmitter Release in Vivo, C. D. Marsden (Ed.), Wiley, New York (1984).

49. S. Cosnier, C. Innocent, L. Allien, S. Poitry, M. Tsacopoulos. Anal. Chem. 69, 968-971 (1997).

50. M. Boutelle, L. Fellows, C. Cook. Anal. Chem. 64, 1790-1794 (1992).

51. P. Lindroth and K. Mopper. Anal. Chem. 51, 1667-1674 (1979).

52. L. Zecca, F. Zambotti, N. Zonta, J. Mantegazza. J. Chromatogr. 233, 307-312 (1982).

53. K. L. Roger, R. A. Philibert, A. J. Allen, J. Molitor, E. J. Wilson, G. R. Dutton. J. Neurosci. Meth. 22, 173-179 (1987).

54. W. Caudill, G. Honck, R. M. Wightman. J. Chromatogr. 227, 331-339 (1982).

55. T. Durkin and G. Anderson. J. Chromatogr. 428, 9-13 (1988).

56. P. Wood and D. Cheney. In Neuromethods 3: Amino Acids, A. Boulton and G. Baker (Eds.), Humana Press, Clefton, NJ (1985).

57. J. Ferkany. In Neuromethods 3: Amino Acids, A. Boulton and G. Baker (Eds.), Humana Press, Clefton, NJ (1985).

58. R. Butterworth. In Neuromethods 3: Amino Acids, A. Boulton and G. Baker (Eds.), Humana Press, Clefton, NJ (1985).

59. D. G. Nicholls. J. Neurochem. 52, 331-341 (1989).

60. M. Fosse, J. Kolstad, F. Fonnom. J. Neurochem. 47, 340-349 (1986).

61. B. Dremel, R. Schmidt, O. Wolfbeis. Anal. Chim. Acta 248, 351-358 (1991).

62. S. Kar and M. Arnold. Anal. Chem. 64, 2438-2443 (1992).

63. W. Opdjcke, S. Park, M. Meyerhoff. Anal. Chim. Acta 155, 11-20 (1983).

64. O. Wolfbeis, L. Weis, M. Leiner. Anal. Chem. 60, 2028-2035 (1988).

65. R. Villarata, D. Cunningham, G. Guilbault. Talanta 38, 49-60 (1991).

66. T. Yao, N. Kobayashi, T. Wasa. Anal. Chim. Acta 231, 121-135 (1990).

67. G. Diaz, L. El-Issa, M. Arnold, R. Miller. J. Neurosci. Meth. 23, $63-67$ (1988).

68. Z. Wang and E. S. Yeung. Appl. Spectrosc. 53, 1502-1506 (1999).

69. Z. Wang, P. G. Haydon, E. S. Yeung. Anal. Chem. 72, 2001-2007 (2000).

70. W. A. Bridger and J. F. Henderson. Cell ATP, Wiley, New York (1983). 
71. B. Alberts, D. Bray, J. Lewis, M. Raff, K. Roberts, J. Watson. Molecular Biology of the Cell, pp. 43-91, 483-549, Garland, New York (1983).

72. A. Lundin and I. Styrelius. Clin. Chim. Acta 87, 199-209 (1978).

73. A. Lundin, U. Karnell, M. Baltscheffsky. Acta. Chem. Scand. B33, 609-609 (1979).

74. A. Bianchi, A. Domenech, E. Garcia-Espana. Anal. Chem. 65, 3137-3142 (1993).

75. P. Singhal and W. G. Kuhr. Anal. Chem. 69, 3552-3557 (1997).

76. A. Barth, K. Hauser, W. Mantele. J. Amer. Chem. Soc. 117, 10311-10316 (1995).

77. P. Stanley, B. McCarthy, R. Smither. ATP Luminescence, Blackwell, Oxford (1989).

78. N. Imbeault, M. Paquet, R. Cote. J. Water Qual. Res. Canada 33, $403-415$ (1998).

79. E. Abraham, P. Okunieff, S. Scala, P. Vos, M. Oosterveld, A. Chen, B. Shrivastav. Science 275, 1324-1326 (1997).

80. B. Bitler and W. McElroy. Arch. Biochem. Biophys. 72, 358-368 (1957).

81. J. Denburg, L. Reiko, W. McElroy. Arch. Biochem. Biophys. 134, 381-394 (1969).

82. W. Rhodes and W. McElroy. J. Biol. Chem. 233, 1528-3157 (1958).

83. R. Schifman, M. Wieden, J. Broker, M. Chery, M. Delduca, K. Norgard, C. Palen, N. Reis, J. Swanson, J. White. J. Clin. Microbiol. 20, 644-648 (1984).

84. O. Molin and L. Nilsson. J. Clin. Microbiol. 18, 521-525 (1983).

85. M. Deluca and W. McElroy (Eds.). In Bioluminescence and Chemiluminescence, Academic Press, New York (1981).

86. R. Dadoo, A. Seto, L. Colon, R. Zare. Anal. Chem. 66, 303-306 (1994).

87. B. Beckers, H. Lang, D. Schimke, A. Lammers. Euro. J. Clin. Microbiol. 4, 556-561 (1985).

88. D. Bussey and K. Tsuji. Appl. Environ. Microbiol. 51, 349-355 (1986).

89. R. Daigneault, A. Larouche, G. Thibault. Clin. Chem. 25, 1639-1643 (1979).

90. R. Hamilton and O. Holm-Hansen. Lumin. Oceanogr. 12, 319-324 (1967).

91. N. Macleod and E. Chappelle, A. Crawford. Nature 223, (1969).

92. G. Levin. Adv. Appl. Microbiol. 10, 55-71 (1968).

93. T. Olsson, K. Sandsted, O. Holmberg, A. Thore. Biotech. Appl. Biochem. 8, 361-369 (1986).

94. R. Bobbin and M. Thompson. Ann. Otol. Rhinol. Laryngol. 87, 185-190 (1978).

95. B. Mockett, G. Housley, P. Thorne. J. Neurosci. 14, 6992-7007 (1994).

96. J. Ashmore and H. Ohmori. J. Physiol. (London) 428, 109-131 (1990).

97. X. Lin, R. Hume, A. Nuttall. J. Neurophysiol. 70, 1593-1605 (1993).

98. D. Dulon, P. Mollard, J. Aran. J. Neuroreport. 2, 69-72 (1991).

99. T. Yamasita, H. Amano, T. Kumazawa. ORL J. Otorhinolaryngol. Relat. Spec. 55, 201-204 (1993).

100. P. Wangemann. Auditory Neurosci. 2, 187-192 (1996).

101. A. Wieraszko, G. Goldsmith, T. Seyfried. Brain Res. 485, 244-250 (1989).

102. E. M. Silinsky and R. S. Redman. J. Physiol. 492, 815-822 (1996).

103. R. S. Redman, E. M. Silinsky. J. Physiol. 477, 117-127 (1994).

104. N. Morel and F. Meunier. J. Neurochem. 36, 1766-1773 (1981).

105. J. Sawynok, J. Downie, A. Reid, C. Cahill, T. White. Brain Res. 610, 32-38 (1993).

106. V. Cena and E. Rojas. Biochim. Biophys. Acta 1023, 213-222 (1990).

107. G. Burnstock and C. Kennedy. Gen. Pharmacol. 16, 433-440 (1985).

108. R. T. Kennedy, R. L. St. Claire, J. G. White, J. W. Jorgenson. Mikrochim. Acta II, 37-45 (1987).

109. R. A. Wallingford and A. G. Ewing. J. Chromatogr. 441, 299-309 (1988).

110. T. M. Olefirowicz and A. G. Ewing. Anal. Chem. 62, 1872-1876 (1990).

111. T. M. Olefirowicz and A. G. Ewing. Chimia 45, 106-108 (1991).

112. B. L. Hogan and E. S. Yeung. Anal. Chem. 64, 2841-2845 (1992). 